\title{
Desigualdad en mortalidad infantil entre las comunas de la provincia de Santiago
}

\author{
Enrique Donoso S.
}

\author{
Inequalities in infant mortality \\ in Santiago
}

Background: Social and economical inequalities have an adverse effect on infant mortality. Aim: To test if the poorest communities of Santiago have the highest rates of infant mortalilty. Material and methods: Variables were obtained from the year 2000 Vital Statistics yearbook and the National Socioeconomic Characterization inquiry. Infant mortality was correlated with the mean income of households, the population below the threshold of poverty and the unemployed population of the 32 municipalities of the Santiago Province. The ratio and the difference in mortality rates between the communities with the higher and lower incomes and the attributable population risk for infant mortality in the Province of Santiago was calculated. Results: Infant mortality was positively correlated with the population below the threshold of poverty ( $\mathrm{r}=0.383 ; \mathrm{p}=0.03$ ) and the unemployed population $(\mathrm{r}=0.437 ; \mathrm{p}=0.012)$ and inversely correlated with the mean household income $(\mathrm{r}=0.522 ; \mathrm{p}=0.002)$. Infant mortality in the poorest community was 2.2 times higher than in the richest one. The difference in rates was 6.6/1000 live births. The attributable population risk determined that it is possible to reduce infant death by $57.8 \%$. Conclusions: In the Province of Santiago, the poorest communities have the highest infant mortality (Rev Méd Chile 2004; 132: 461-6).

(Key Words: Income; Infant mortality; Poverty areas)

Recibido el 17 de julio, 2003. Aceptado en versión corregida el 13 de enero, 2004.

Departamento de Obstetricia y Ginecología, Facultad de Medicina, Pontificia Universidad Católica de Chile. Santiago de Chile.

Las as desigualdades sociales y económicas, tanto en países desarrollados como en vías de desarrollo, afectan adversamente la salud de los más vulnerables, especialmente a los niños en su primer año de vida ${ }^{1-3}$. La tasa de mortalidad infantil es un indicador que resume la calidad de la infraestructura sanitaria de un país, el efecto del proceso reproductivo y de las condiciones am-

Correspondencia a: Dr. Enrique Donoso S. Alameda 340. Casilla 114-D. Santiago, Chile. Fax: 6331457. E mail: edonoso@med.puc.cl bientales, sociales y económicas que pueden actuar negativamente sobre la salud del niño. Chile redujo la mortalidad infantil, desde 119,5/ 1.000 nacidos vivos en 1960 a 8,9 en $2000^{4,5}$, ubicando al país en lugares de privilegio dentro de Latinoamérica ${ }^{6}$. Sin embargo, las cifras promedios, por auspiciosas que sean, no miden adecuadamente las desigualdades en salud $y$, habitualmente, ocultan disparidades entre los diferentes sectores que forman una población. La mortalidad infantil en Chile, en el período 19851999, se asoció significativamente a indicadores 
económicos como el producto interno bruto, el producto interno bruto per cápita, el índice real de salario mínimo, el desempleo y la inflación anual $^{7}$, esto sugiere que los grupos de nivel socioeconómico bajo podrían tener tasas mayores de mortalidad infantil que los grupos de nivel socioeconómico más alto.

Se postula que en la provincia de Santiago, las comunas con mayor pobreza tienen tasas de mortalidad infantil más altas.

\section{MATERIAL Y MÉTODO}

Se investigó la desigualdad en mortalidad infantil entre las 32 comunas que forman la provincia de Santiago en 2000, mediante la correlación de la tasa de mortalidad infantil con el ingreso promedio mensual de los hogares, la población bajo la línea de pobreza y la población desocupada. El análisis de desigualdad en mortalidad infantil se efectuó según metodología recomendada por la Organización Panamericana de la Salud ${ }^{3,8}$, mediante la razón de las tasas de mortalidad infantil (TMI), la diferencia de las tasas de mortalidad infantil y el riesgo atribuible poblacional (RAP) de mortalidad infantil en la provincia de Santiago mediante la fórmula siguiente:

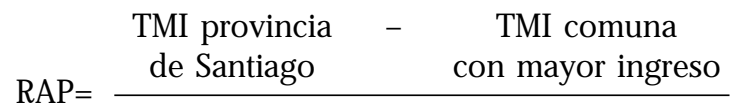

TMI provincia de Santiago

La razón de las tasas de mortalidad infantil es el cuociente entre las tasas de la comuna con menor y mayor ingreso promedio mensual de los hogares, esto permite calcular cuántas veces más mortalidad infantil hay entre esas comunas. La diferencia de las tasas de mortalidad infantil es la resta entre las tasas de la comuna con menor y mayor ingreso promedio mensual de los hogares, esa diferencia relacionada al número de nacidos vivos de la comuna con menor ingreso permite calcular en ella el exceso de muertes infantiles. El cálculo del riesgo atribuible poblacional permite estimar la reducción porcentual y absoluta de mortalidad infantil en la provincia de Santiago, si todas las comunas tuvieran la TMI de la comuna con mayor ingreso promedio mensual de los hogares.
La asociación entre la mortalidad infantil y las variables socioeconómicas se efectuó según análisis de correlación de Pearson y la comparación entre proporciones con la prueba de $\chi^{2}$ con corrección de Yates. Se estableció como nivel de significación estadística un $\mathrm{p}<0,05$.

Los conceptos de nacido vivo y de mortalidad infantil son los internacionalmente aceptados. Los nacidos vivos (NV) y las muertes infantiles se registran con relación a la comuna de residencia habitual de la madre, información obtenida del Instituto Nacional de Estadísticas ${ }^{5}$. Se calculan las tasas de mortalidad infantil por comunas, expresadas por 1.000 nacidos vivos ocuridos e inscritos en 2000. Las variables socioeconómicas por comunas, se obtienen de la encuesta de Caracterización Socioeconómica Nacional $(\mathrm{CASEN})^{9}$, que definió población bajo la línea de pobreza aquella con un ingreso mínimo individual de $\$ 40.562$ de noviembre de 2000 y como desocupados a la población mayor de 15 años que en los últimos 2 meses hicieron esfuerzos concretos para encontrar trabajo. El ingreso promedio mensual de los hogares se expresa en su equivalencia en dólares americanos al 30 de diciembre de 2000 (1 US $\$=572,28$ pesos chilenos).

\section{RESUlTADos}

En la provincia de Santiago en 2000, hubo 78.328 nacidos vivos ocurridos e inscritos y 1.038 muertes de menores de 1 año y una tasa de mortalidad infantil de 13,3/1.000 NV.

El rango de los nacimientos estuvo determinado por Maipú (7.799 NV) y Lo Barnechea (1.078 $\mathrm{NV}$ ), las muertes infantiles por Maipú (126 muertes) y La Cisterna (5 muertes) y las tasas de mortalidad infantil por Lo Espejo (19,7/1.000 NV) y La Cisterna (4,0/1.000 NV) (Tabla 1).

El rango de ingreso promedio mensual de los hogares fue dado por Lo Barnechea (US\$4.781,6) y La Pintana (US\$481,1), la población bajo la línea de la pobreza por Providencia (0\%) y La Pintana $(31,1 \%)$ y la población desocupada por Renca (20,2\%) y Vitacura (2,9\%) (Tabla 2).

Los valores de tendencia central y de dispersión, muestran la gran amplitud de la distribución de las variables estudiadas, especialmente del ingreso promedio mensual de los hogares, con un índice de asimetría de +2,077 (Tabla 3). 
Tabla 1. N acidos vivos (N V), muertes infantiles (MI) y tasa de mortalidad infantil (T MI), de las comunas de la provincia de Santiago. Chile, 2000

\begin{tabular}{|lrrr|}
\hline Comunas & NV & MI & TMI \\
\hline Cerrillos & 1.107 & 9 & 8,1 \\
Cerro Navia & 2.703 & 32 & 11,8 \\
Conchalí & 2.421 & 31 & 12,8 \\
El Bosque & 3.148 & 46 & 14,6 \\
Estación Central & 2.156 & 36 & 16,7 \\
Huechuraba & 1.595 & 22 & 13,8 \\
Independencia & 1.182 & 12 & 10,2 \\
La Cisterna & 1.256 & 5 & 4,0 \\
La Florida & 5.898 & 76 & 12,9 \\
La Granja & 2.554 & 35 & 13,7 \\
La Pintana & 3.772 & 46 & 12,2 \\
La Reina & 1.284 & 18 & 14,1 \\
Las Condes & 3.647 & 41 & 11,3 \\
Lo Barnechea & 1.078 & 6 & 5,6 \\
Lo Espejo & 2.030 & 40 & 19,7 \\
Lo Prado & 1.897 & 35 & 18,4 \\
Macul & 1.737 & 18 & 10,3 \\
Maipú & 7.799 & 126 & 16,2 \\
Nuñoa & 2.135 & 29 & 13,6 \\
Pedro Aguirre Cerda & 1.774 & 26 & 14,7 \\
Peñalolén & 4.041 & 52 & 12,9 \\
Providencia & 1.613 & 12 & 7,5 \\
Pudahuel & 3.242 & 41 & 12,6 \\
Quilicura & 2.093 & 26 & 12,4 \\
Quinta Normal & 1.781 & 25 & 14,0 \\
Recoleta & 2.744 & 38 & 13,9 \\
Renca & 2.316 & 33 & 14,3 \\
San Joaquín & 1.533 & 18 & 11,8 \\
San Miguel & 1.160 & 17 & 14,7 \\
San Ramón & 2.167 & 32 & 14,8 \\
Santiago & 3.269 & 47 & 14,4 \\
Vitacura & 1.196 & 8 & 6,7 \\
\hline
\end{tabular}

Fuente: Instituto Nacional de Estadísticas ${ }^{5}$.
Tabla 2. Caracterización socioeconómica de las comunas de la Provincia de Santiago. Chile, 2000

\begin{tabular}{|lrrr|}
\hline Comunas & $\begin{array}{r}\text { Ingreso } \\
\text { (US\$) }\end{array}$ & $\begin{array}{c}\text { Pobreza } \\
(\%)\end{array}$ & $\begin{array}{c}\text { Desocupados } \\
(\%)\end{array}$ \\
\hline Cerrillos & & & \\
Cerro Navia & 783,1 & 9,7 & 12,7 \\
Conchalí & 566,6 & 23,9 & 16,8 \\
El Bosque & 836,9 & 18,5 & 12,8 \\
Estación Central & 649,1 & 21,5 & 14,5 \\
Huechuraba & 796,9 & 12,8 & 9,5 \\
Independencia & $1.127,8$ & 21,4 & 10,2 \\
La Cisterna & 996,3 & 10,5 & 8,8 \\
La Florida & $1.073,6$ & 12,8 & 6,3 \\
La Granja & $1.043,8$ & 8,5 & 10,1 \\
La Pintana & 589,3 & 22,0 & 18,9 \\
La Reina & 481,1 & 31,1 & 15,2 \\
Las Condes & $2.793,5$ & 3,6 & 5,0 \\
Lo Barnechea & $4.216,9$ & 0,2 & 4,7 \\
Lo Espejo & $4.781,6$ & 8,5 & 4,1 \\
Lo Prado & 517,4 & 27,0 & 15,7 \\
Macul & 675,9 & 14,8 & 13,2 \\
Maipú & 941,1 & 13,7 & 9,8 \\
Ñuñoa & 782,5 & 7,4 & 6,6 \\
Pedro Aguirre Cerda & $2.003,2$ & 2,3 & 5,7 \\
Peñalolén & 938,0 & 15,7 & 8,2 \\
Providencia & $2.831,5$ & 15,7 & 8,8 \\
Pudahuel & 719,4 & 18,7 & 5,9 \\
Quilicura & 743,5 & 16,0 & 9,9 \\
Quinta Normal & 765,8 & 18,3 & 9,8 \\
Recoleta & 804,4 & 15,3 & 11,2 \\
Renca & 590,9 & 25,7 & 20,2 \\
San Joaquín & 744,8 & 15,7 & 10,5 \\
San Miguel & $1.219,8$ & 8,2 & 9,6 \\
San Ramón & 649,6 & 29,0 & 13,2 \\
Santiago & $1.212,3$ & 7,9 & 8,9 \\
Vitacura & $4.329,6$ & 0,7 & 2,9 \\
\hline
\end{tabular}

Fuente: CASEN, $2000^{9}$.

Tabla 3. Valores de tendencia central y de dispersión de las variables sanitarias y socioeconómicas de las comunas de la Provincia de Santiago

\begin{tabular}{|lrrrrrrr|}
\hline Variables & Mínimo & Máximo & Media & Mediana & \pm DE & CV & Asimetría* \\
\hline TMI (x1000 NV) & 4,0 & 19,7 & 12,6 & 13,2 & 3,43 & 0,27 & $-0,646$ \\
Ingreso (US\$) & 481,1 & $4.781,6$ & $1.308,7$ & 800,6 & $1.168,6$ & 0,89 & $+2,077$ \\
Pobreza (\%) & 0,0 & 31,1 & 14,2 & 15,1 & 8,39 & 0,59 & $+0,075$ \\
Desocupados (\%) & 2,9 & 20,2 & 10,3 & 9,8 & 4,22 & 0,40 & $+0,425$ \\
\hline
\end{tabular}

TMI: tasa de mortalidad infantil. *Skewness. DE: desvío estándar. CV: coeficiente de variabilidad. 
La mortalidad infantil se correlacionó directa y significativamente con la población bajo la línea de pobreza $(\mathrm{R}=+0,383 ; \mathrm{p}=0,03)$ y de desocupados $(R=+0,437 ; p=0,01)$, e inversamente con el ingreso promedio mensual de los hogares $(R=-0,522$; $\mathrm{p}=0,002$ ) (Tabla 4).

La mortalidad infantil de las comunas con ingreso promedio mensual de los hogares igual 0 superior a US\$4.000, no presentó diferencias significativas con las en rangos de US\$2.000-3.999 $(p=0,247)$ y de US $\$ 1.000-1.999(p=0,052)$, mientras que fue significativamente inferior a las en rango de US\$700-999 $(p=0,008)$ y menor a US\$700 $(\mathrm{p}=0,002)$ (Tabla 5).

La razón de las tasas de mortalidad infantil entre la comuna de menor y mayor ingreso promedio mensual de los hogares mostró que en La Pintana (12,2/1.000 NV) hubo 2,2 veces más mortalidad infantil que en Lo Barnechea $(5,6 /$ $1.000 \mathrm{NV}$ ), con una diferencia entre sus tasas de $6,6 / 1.000 \mathrm{NV}$, equivalente a un exceso de 25 muertes infantiles anuales. El riesgo atribuible poblacional de mortalidad infantil para la provincia de Santiago fue 57,8\% equivalente a un exceso de 599 muertes infantiles anuales, que es posible reducir si todas las comunas tuvieran la tasa de mortalidad infantil de Lo Barnechea.

\section{DisCUSIÓN}

Este estudio demostró que, en la Provincia de Santiago, las variables socioeconómicas analizadas se asocian significativamente a mortalidad infantil,
Tabla 4. Correlación entre la mortalidad infantil con las variables socioeconómicas de las comunas de la provincia de Santiago

\begin{tabular}{|ll|}
\hline $\begin{array}{l}\text { Variables } \\
\text { socioeconómicas }\end{array}$ & Correlación \\
\hline Ingreso (US\$) & $\mathrm{R}=-0,522 ; \mathrm{p}=0,002$ \\
Pobreza (\%) & $\mathrm{R}=+0,383 ; \mathrm{p}=0,03$ \\
Desocupados (\%) & $\mathrm{R}=+0,437 ; \mathrm{p}=0,01$ \\
\hline
\end{tabular}

esto explica que las comunas con mayor pobreza tengan tasas de mortalidad infantil más alta.

Así, la comuna más pobre (La Pintana) tuvo 2,2 veces más mortalidad infantil que la más rica (Lo Barnechea) y comunas como Vitacura, Providencia y Lo Barnechea, con ingreso promedio mensual de los hogares superiores a US $\$ 2.800$, tuvieron tasas de mortalidad infantil equivalentes a la de países desarrollados ${ }^{10}$, mientras que Maipú, Estación Central, Lo Prado y Lo Espejo, con ingreso promedio mensual de los hogares inferiores a US\$800 presentaron tasas que tenía Chile entre 1984 y 19905. Es importante destacar que el ingreso promedio mensual de los hogares inferior a US\$1.000 establece diferencias significativas en la tasa de mortalidad infantil y que la mediana en la provincia de Santiago es inferior a ese punto de corte (US\$800), por lo que mejorar las condiciones sociales y económicas de la población más pobre permitirá reducir la brecha en mortalidad infantil ${ }^{11}$. La importante asimetría del ingreso promedio mensual de los hogares de

Tabla 5. Tasas de mortalidad infantil promedio (T MI) según rangos de ingreso promedio mensual de los hogares de las comunas de la provincia de Santiago

\begin{tabular}{|lccc|}
\hline $\begin{array}{l}\text { Ingreso } \\
\text { (US\$) }\end{array}$ & $\begin{array}{c}\text { Nacidos vivos } \\
(\mathrm{n})\end{array}$ & $\begin{array}{c}\text { Muertes infantiles } \\
(\mathrm{n})\end{array}$ & $\begin{array}{c}\text { TMI } \\
(\mathrm{x} 1000 \mathrm{NV})\end{array}$ \\
\hline$\geq 4.000$ & 5.921 & 55 & 9,3 \\
$2.000-3.999$ & 5.032 & 59 & $11,7 *$ \\
$1.000-1.999$ & 13.178 & 167 & $12,7^{*}$ \\
$700-999$ & 31.836 & 432 & $13,6^{* *}$ \\
$<700$ & 22.361 & 325 & $14,5^{* *}$ \\
\hline
\end{tabular}

* Diferencia no significativa con los grupos de ingreso promedio $\geq$ US $\$ 4.000$.

** Diferencia significativa con los grupos de ingreso promedio $\geq \mathrm{US} \$ 4.000$. 
la provincia de Santiago, determinó que el riesgo atribuible poblacional de mortalidad infantil fuese de $57,8 \%$ y representa la capacidad de reducción que tiene la provincia si todas sus comunas alcanzaran la tasa de mortalidad infantil de Lo Barnechea.

La interpretación de estos resultados exige cautela. Los estudios ecológicos se realizan en grupos poblacionales, de ahí que los resultados no puedan ser traspasados al plano individual, ya que se caería en la denominada falacia ecológi$\mathrm{ca}^{12}$. Desigualdad no es sinónimo de inequidad, esta última es una desigualdad injusta y evitable, pero estos conceptos se acercan cuando los organismos responsables de la salud de una sociedad no ejercen las acciones destinadas a disminuir la brecha entre las personas que la forman ${ }^{13}$.

En Chile, a fines de 2000, 20,6\% (3.081.000 personas) de la población estaba en situación de pobreza, que correspondía al 16,6\% de los hogares (643.200 hogares). Sin embargo, en la década 1990-99, hubo una significativa reducción de la

\section{REFERENCIAS}

1. León DA, Vagero D, Olausson PO. Social class differences in infant mortality in Sweden: comparison with England and Wales. BMJ 1992; 305: 687-91.

2. BiRd ST, BaUmann KE. State-level infant, neonatal and postneonatal mortality: the contribution of structural socioeconomic variable. Int J Health Serv 1998; 28: 13-27.

3. CastiLo-Salgado C, Loyola E, Roca A. Desigualdades en la Mortalidad Infantil en la Región de las Américas: Elementos básicos para el análisis. Boletón Epidemiológico/OPS 2001; 22: 4-7.

4. TAUCHER E, JofrÉ I. Mortalidad infantil en Chile: el gran descenso. Rev Méd Chile 1997; 125: 1225-35.

5. Instituto Nacional de Estadísticas. Anuario de Estadísticas Vitales. Chile, 2000.

6. UNICEF. Latin America and the Caribbean. Statistical data. Disponible en: www.unicef.org/statis/ latinamerica.html. Acceso el 6 de junio de 2003.

7. Szot J. Mortalidad infantil e indicadores económicos en Chile: 1985-1999. Rev Méd Chile 2002; 130: $107-12$. pobreza de $38,6 \%$ en 1990 a $20,6 \%$ en 2000 , producto del mayor gasto fiscal en programas sociales $^{14}$, paralelamente, en ese período la mortalidad infantil descendió de 16,0 a 8,9/1.000 NV55.

En Latinoamérica, Chile, Cuba y Costa Rica, han demostrado que, a través de una buena organización del sistema de salud, es posible reducir la mortalidad infantil junto con mejorar las condiciones sociales de la población; así, en el período 1990-2000, Chile redujo la mortalidad infantil de 16,0 a 8,9/1.000 NV (44,3\%), Cuba de 10,7 a $7,2 / 1.000 \mathrm{NV}(32,7 \%)$ y Costa Rica de 14,7 a 10,2/1.000 NV (30,6\%)

El análisis de las causas de mortalidad infantil y de los factores condicionantes permiten diseñar, mantener y fortalecer las estrategias de salud pública destinadas a su reducción ${ }^{18}$, las que junto al desarrollo social, cultural y económico de los grupos de menores recursos permitirán estrechar la brecha de desigualdad en salud infantil en Chile ${ }^{19}$.

Se concluye que en la provincia de Santiago las comunas con mayor pobreza tienen tasas de mortalidad infantil más altas.

8. Schneider MC, Castillo-Salgado C, Bacallao J, Loyola E, Mujica OJ, Vidaurre M et al. Métodos de medición de las desigualdades de salud. Rev Panam Salud Pública 2002; 12: 398-414.

9. Ministerio de Planificación. Caracterización Socioeconómica Nacional (CASEN). Chile, 2000. Disponible en: www.casen.cl. Acceso el 6 de junio de 2003.

10. MacDorman MF, Minino AM, Strobino DM, Guyer B. Annual Summary of Vital Statistics - 2001. Pediatrics 2002; 110: 1037-52.

11. Ministerio de Planificación. Impacto distributivo del gasto social. Informe ejecutivo, 2000. Santiago de Chile, julio 2001. Disponible en: www.mideplan.cl. Acceso el 6 de junio de 2003.

12. Greentan S, Morgenstern H. Ecological bias, confounding and effect modification. Int J Epidemiol 1989; 18: 269-74.

13. International Society for Equity in Health. Toronto Declaration on Equity in Health. Toronto, June 2002. Disponible en: www.iseqh.org/en/ index.htm. Acceso el 6 de junio de 2003.

14. Ministerio de Planificación. Pobreza e indigencia e impacto del gasto social en la calidad de 
vida. Santiago de Chile, julio 2001. Disponible en: www.mideplan.cl. Acceso el 6 de junio de 2003.

15. Riverón CoRTeguera RL. Estrategias para reducir la mortalidad infantil, Cuba 1959-1999. Rev Cubana Pediatr 2000; 72: 147-64.

16. InFomed. Anuario Estadístico de Salud, Cuba 2000. Disponible en: www.sld.cu/servicios/ estadisticas/. Acceso 30 de septiembre de 2003.
17. Instituto Nacional de Estadísticas y Censos de Costa Rica. Mortalidad infantil y Evolución Reciente, 2002. Disponible en: www.inec.go.cr. Acceso 30 de septiembre de 2003.

18. KAEMPFFER MA, MEDINA E. Análisis de la mortalidad infantil y factores condicionantes. Chile, 1998. Rev Chil Pediatr 2000; 71: 405-12.

19. Ministerio de Salud. Objetivos sanitarios y modelo de salud para la década 2000-2010. Documento Oficial. Gobierno de Chile. Enero de 2000. 